\title{
Effect of Extraction Methods on the Types and Levels of Free Amino Acid of Beef Longissimus Muscle
}

\author{
Dashmaa Dashdorj and InHo Hwang* \\ Department of Animal Science and Institute of Rare Earth for Biological Application, \\ Chonbuk National University, Jeonju 561-756, Korea
}

\begin{abstract}
The current study was carried out to investigate the impact of extraction conditions on the free amino acid level and type in beef longissimus muscle. The sample blocks were chiller aged for $1 \mathrm{~d}$ and $7 \mathrm{~d}$ at $4^{\circ} \mathrm{C}$. There are three homogenization speeds $(11,000,19,000$ and 24,000 rpm) for bigger and two speeds (11,000 and 13,000 rpm) for smaller homogenizer's dispersing tools were used for evaluation. Results showed that chiller ageing greatly $(p<0.05)$ increased extractable free amino acids, except cystine. Homogenization with the bigger dispersing tool at 24,000 rpm resulted in the highest free amino acid levels for both 1 and $7 \mathrm{~d}$ samples. Significant differences $(p<0.05)$ in the mean values of most amino acids due to the effect of speed and interactions between ageing times. However, the speed effect and interaction between ageing with homogenization speed were not significant $(p>0.05)$ for most of the amino acids except valine and isoleucine when using the smaller dispensing tools. The current data indicated that a standardized method for free amino acid types and levels of aged beef samples. In addition, the results also suggested that utilization of a big dispensing tool at high homogenization speed is a better condition for releasing free amino acid contents in beef samples.
\end{abstract}

Key words: free amino acids, beef, homogenization effect, ageing, speed, dispensing tools

\section{Introduction}

Meat is considered as the high-quality protein source because of their balances in amino acid contents, especially all the essential amino acids necessary for physical and mental well-being. On the other hand, free amino acids also contribute to meat taste and indirectly to aroma flavors by generation of volatile compounds through the Maillard reactions and Strecker degradations (Aristoy and Toldra, 2009).

The amino acid profiles can be viewed as a two-stage process. The first stage involves the sample preparation and the second stage involves chromatographic analysis. The homogenization treatment is a fundamental step of the analysis because the successful determination of free amino acids is dependent on the efficient disruption and homogenization of muscle tissues (Kivi, 2000). The efficient disruption facilitates the lysis of the starting material and release all the free amino acids contained in the meat

*Corresponding author: InHo Hwang, Department of Animal Science and Institute of Rare Earth for Biological Application, Chonbuk National University, Jeonju 561-756, Korea. Tel: 82-63270-2605, Fax: 82-63-270-2605, E-mail: inho.hwang@chonbuk. ac.kr sample, ensuring high yield of amino acids. Incomplete disruption may results in reduced yields. Earlier studies indicated that the homogenization conditions result in different free amino acids level as a consequence of various disruption impacts. The shaft homogenizer-rotor stators is one of the most widely used tools for animal tissues (Burden, 2008), which generated the smallest fragments than CryoGrinder and Omni mixer (Hopkins et al., 2004). In addition, the speed and duration of homogenization may greatly interact with homogenizer types (Hopkins et al., 2004), otherwise the speed and efficiency of homogenization were greatly eroded when a smaller homogenizer was adopted (Burden, 2008).

Most of the laboratory rotor stator homogenizer's dispersers which allow, for example, keeping track of the speed range up to $27,000 \mathrm{rpm}$. In the case of dispersers, stainless steel dispersing elements are capable of processing between 0.03 and $2 \mathrm{~L}$ of samples with 5 to $25 \mathrm{~mm}$ diameter interchangeable generator probes. Sample volumes can range from $0.25 \mathrm{~mL}$ to $1,000 \mathrm{~mL}$ with the available of different size. For consistency results of free amino acid levels in beef over ageing period, it is very important to choose a right generator and dispersers for fibrous tissue samples which matches your sample volume and 
required level of dispersion. Also, the selection of homogenizer type, tools such as dispersing elements and homogenization method may have a significant impact on the outcome (Halpine, 2004). However most cases the type of homogenizer, speed and dispersing elements has not been specified in the published papers (Hopkins et al., 2004). These together can result in large variations in free amino acids content between laboratories and experiments and thus consequently generating controversial data.

The objective of this study was to evaluate the effects of a sampling technique particularly homogenization on the types and levels of amino acids for differently aged beef sample. The free amino acid contents as a function of the extraction condition were assessed using a HPLC technique equipped with a diode array detector (DAD) detector. The current study emphasized the importance of standard method for consistent results and compatible data with other laboratories.

\section{Materials and Methods}

\section{Chemicals}

HPLC-grade purified water, acetonitrile and methanol were purchased from J.T Baker (USA), amino acid standards, extended amino acids, also derivatization reagents; FMOC, OPA reagent, borate buffer were purchased from Agilent technologies (Waldbronn, Germany). Triethylamine was purchased from Fluka (Belgium), sodium phosphate and hydrochloric acid was purchased from Merck (Darmstadt, Germany). Sodium tetraborate decahydrate, sodium azide and ortho phosphoric acid were purchased from Sigma (Steinneim, Germany), filter papers were from Whatman (Maidstone, England) and membrane PTFE (pore: $0.45 \mu \mathrm{m}$, diameter: $13 \mathrm{~mm}$ ) was obtained from Sartorius (Goettingem, Cermany).

\section{Experimental design}

Muscle sampling and extraction of free amino acids

M. longissimus (LD) muscles of Hanwoo beef was sampled from a commercial slaughter house day after slaughter and aged for $7 \mathrm{~d}$ at $4^{\circ} \mathrm{C}$. All samples were frozen at $-40^{\circ} \mathrm{C}$ until analyzed for both experiment 1 and 2. Sample preparation for the detection of free amino acid was performed by Aristoy and Toldra (1991) with suitable modification as described by Hwang et al. (2004).

\section{Experiment 1}

Briefly, approximately $1 \mathrm{~g} \mathrm{LD}$ muscle tissue was homogenized in $5 \mathrm{~mL}$ of $0.01 \mathrm{~N} \mathrm{HCl}$ of $50 \mathrm{~mL}$ conical tube using a rotor-stator homogenizer (Ultra turrax T 25 basic, Ika, Malaysia) with bigger S25 NK 19 G dispersing tool at low $(11,000 \mathrm{rpm})$, medium $(19,000 \mathrm{rpm})$ or high $(24,000$ rpm) for three strokes of $20 \mathrm{~s}$ each.

\section{Experiment 2}

For the smaller S8 N8G dispersing tools, the samples were homogenized at low speed $(11,000 \mathrm{rpm})$ and high $(13,000 \mathrm{rpm})$ for three strokes of $20 \mathrm{~s}$ each. The homogenized samples were then centrifuged at $10,000 \mathrm{~g}$ for 20 $\min$ at $4^{\circ} \mathrm{C}$. The supernatant was filtered through $541 \#$ Whatman filter paper and stored at $-80^{\circ} \mathrm{C}$ until use. $10 \mu \mathrm{L}$ of ISTD stock solution ( $1 \mathrm{nmol}$ norvaline) was added into $300 \mu \mathrm{L}$ thawed samples and left for $30 \mathrm{~min}$ at room temperature followed deproteinized with $690 \mu \mathrm{L}$ of acetonitrile then centrifuged at $10,000 \mathrm{~g}$ for $15 \mathrm{~min}$. Two hundred micro liters of the supernatant were used for derivative step. Each analysis was done in triplicates

\section{Instrumental analysis}

The free amino acid contents in beef sample from experiment 1 and 2 were analyzed using a RP-HPLC 1200 system (Agilent Technologies Inc., USA) equipped with a diode array detector (DAD). HPLC conditions for free amino acids followed as described by Woodward and Henderson (2007). A rapid Resolution HT Eclipse XDBC18 column ( $4.6 \mathrm{~mm} \times 150 \mathrm{~mm}$ column packed with $5 \mu \mathrm{m}$ particles Agilent Technologies Inc., USA) was used for separation of amino acids. Two hundred microlitres of the supernatant were derivatised with OPA for the primary amino acids and FMOC for the secondary amino acids; furthermore a $0.4 \mathrm{~N}$ borate buffer was used. Briefly, OPA-derivatised amino acids were monitored at $338 \mathrm{~nm}$ and FMOC-derivatised amino acids were monitored at $262 \mathrm{~nm}$. The column temperature at $40^{\circ} \mathrm{C}$; flow rate was $2 \mathrm{~mL} / \mathrm{min}$, using a gradient between two solvents. Solvent $A$ was $10 \mathrm{mM} \mathrm{Na}_{2} \mathrm{HPO}_{4}: 10 \mathrm{mM} \mathrm{Na}_{2} \mathrm{~B}_{4} \mathrm{O}_{7}: 0.5 \mathrm{mM}$ $\mathrm{NaN}_{3}$ at $\mathrm{pH} 8.2$ and Solvent B was Acetonitrile: Methanol: Water $(45: 45: 10 \mathrm{v} / \mathrm{v})$. The separation was achieved in $8.7 \mathrm{~min}$; linear gradient $2 \% \mathrm{~B}$ for $1 \mathrm{~min}, 2-57 \%$ B for 6 $\min$ and $57-100 \%$ for $0.1 \mathrm{~min}$ then maintain for $1.3 \mathrm{~min}$ and re-equilibrated with $100 \% \mathrm{~A}$ for $0.2 \mathrm{~min}$ before next sample injections. For the calibration curves 17 primary amino acids, plus 4 extended amino acids are combined with fixed amounts of internal standards and final concentration of standard solution were 90,225 and 900 pmol. The primary amino acids were quantified by using external standard procedures or by using norvaline as an internal standard and the secondary amino acids were 
quantified using sarcosine as an internal standard. Individual free amino acid values were expressed as $\mu \mathrm{mol} / \mathrm{g}$. The mechanical shear effect of homogenizing conditions on beef muscle fiber was monitored using an electronic microscope at $40 \times$ magnifications, coupled to a digital camera (Olympus, CKX 41, and Foculus IEEE1394, Germany).

\section{Statistical analysis}

Analysis of variance was performed using the SAS General Linear Models Procedure. Ageing and homogenizer speed and their interaction were presented by least square means nteractions; ageing $\times$ speed. (SAS Institute, Cary, 2007).

\section{Result and Discussion}

Type and level of free amino acids as a function of ageing and speeds of larger dispensing tool

Total of 20 free amino acids were detected in beef samples both aged 1 and $7 \mathrm{~d}$, except hydroxyproline which rarely appears in protein structures. The use of rapid resolution HT Eclipse XDB-C18 column (4.6 mm $\times 150 \mathrm{~mm}$ column with $5 \mu \mathrm{m}$ sized particle) produces a high ana- lytic sensitivity and shortened analytic time, the separation was achieved in $8.7 \mathrm{~min}$ and the total cycle time was approximately $15 \mathrm{~min}$, including injector program prerun DAD auto-balancing, and re-equilibration time.

Changes in free amino acid contents during ageing, using bigger dispensing tool (stator diameter $19 \mathrm{~mm}$ and rotor diameter $12.7 \mathrm{~mm}$ ) and different speed (low-11,000; medium-19,000 and high-24,000 rpm) are shown in Table 1. The alanine $(5.28 \mu \mathrm{mol} / \mathrm{g})$, glutamine $(3.04 \mu \mathrm{mol} / \mathrm{g})$, serine $(0.41 \mu \mathrm{mol} / \mathrm{g})$, valine $(0.32 \mu \mathrm{mol} / \mathrm{g})$ and leucine $(0.49 \mu \mathrm{mol} / \mathrm{g})$ were the most dominant among detected amino acids, while asparagine $(0.14 \mu \mathrm{mol} / \mathrm{g})$ and aspartic acids $(0.1 \mu \mathrm{mol} / \mathrm{g})$ were found to be trace in concentrations in $1 \mathrm{~d}$ aged meat. The mean values of individual free amino acids significantly increased with ageing time except for cystine. An increase in amino acids in beef during ageing period has been elucidated in the previous works in that the proteolysis contributes to the breakdown of the myofibrillar protein, action of calpains and cathepsins on major myofibrillar proteins, generating protein fragments and intermediate size polypeptides, furthermore polypeptides and peptides generate free amino acids (Toldra, 2006). The final protein degradation products, such as peptides and free amino acids were thought to be

Table 1. Changes in free amino acid contents during ageing, and by using bigger homogenizer and different speed $(\mu \mathrm{mol} / \mathrm{g})$

\begin{tabular}{|c|c|c|c|c|c|c|c|c|c|c|}
\hline \multicolumn{11}{|c|}{ Bigger Homogenizer } \\
\hline \multirow{2}{*}{$\begin{array}{l}\text { Amino } \\
\text { acids }\end{array}$} & \multicolumn{3}{|c|}{$1 \mathrm{~d}$} & \multicolumn{3}{|c|}{$7 \mathrm{~d}$} & \multirow{2}{*}{ SEM } & \multirow{2}{*}{ Ageing } & \multirow{2}{*}{ Speed } & \multirow{2}{*}{$\begin{array}{l}\text { Ageing } \\
\times \text { Speed }\end{array}$} \\
\hline & High & Medium & Slow & High & Medium & Slow & & & & \\
\hline ASP & 0.01 & nd & nd & $0.20^{\mathrm{ab}}$ & $0.22^{\mathrm{a}}$ & $0.17^{b}$ & 0.01 & $* * *$ & $*$ & ns \\
\hline GLU & 0.30 & 0.33 & 0.28 & $2.23^{\mathrm{a}}$ & $1.97^{\mathrm{b}}$ & $1.96^{\mathrm{b}}$ & 0.06 & $* * *$ & $*$ & $*$ \\
\hline ASN & $0.14^{\mathrm{a}}$ & $0.07^{b}$ & $0.09^{\mathrm{b}}$ & 0.77 & 0.69 & 0.73 & 0.02 & $* * *$ & $*$ & ns \\
\hline SER & 0.41 & 0.33 & 0.36 & 2.16 & 1.36 & 1.94 & 0.25 & $* * *$ & ns & ns \\
\hline GLN & $3.04^{\mathrm{a}}$ & $1.56^{\mathrm{b}}$ & $2.31^{\mathrm{b}}$ & $8.05^{\mathrm{a}}$ & $6.68^{b}$ & $6.85^{b}$ & 0.37 & $* * *$ & $* *$ & ns \\
\hline HIS & 0.13 & 0.13 & 0.11 & 0.57 & 0.54 & 0.57 & 0.02 & $* * *$ & ns & ns \\
\hline GLY & 0.46 & 0.39 & 0.41 & $1.63^{\mathrm{a}}$ & $1.40^{\mathrm{b}}$ & $1.33^{b}$ & 0.03 & $* * *$ & $* * *$ & $*$ \\
\hline THR & $0.30^{\mathrm{a}}$ & $0.22^{b}$ & $0.22^{b}$ & $1.71^{\mathrm{a}}$ & $1.40^{\mathrm{b}}$ & $1.33^{b}$ & 0.05 & $* * *$ & $* *$ & $*$ \\
\hline $\mathrm{ARG}$ & 0.26 & 0.20 & 0.20 & $1.36^{\mathrm{a}}$ & $0.97^{b}$ & $1.09^{b}$ & 0.03 & $* * *$ & $* * *$ & $* *$ \\
\hline ALA & $5.28^{\mathrm{a}}$ & $5.12^{b}$ & $4.48^{b}$ & $9.10^{\mathrm{a}}$ & $7.48^{b}$ & $6.94^{b}$ & 1.30 & $* *$ & $*$ & $*$ \\
\hline TYR & 0.20 & 0.18 & 0.18 & $1.45^{\mathrm{a}}$ & $0.99^{\mathrm{b}}$ & $1.04^{\mathrm{b}}$ & 0.07 & $* * *$ & $*$ & $*$ \\
\hline CYS & 0.13 & 0.09 & 0.11 & 0.08 & 0.14 & 0.11 & 0.01 & ns & ns & ns \\
\hline VAL & $0.32^{\mathrm{a}}$ & $0.28^{b}$ & $0.24^{b}$ & $1.69^{\mathrm{a}}$ & $1.45^{\mathrm{b}}$ & $1.42^{b}$ & 0.04 & $* * *$ & $* *$ & $*$ \\
\hline MET & 0.17 & 0.14 & 0.13 & $0.77^{\mathrm{a}}$ & $0.71^{b}$ & $0.67^{b}$ & 0.01 & $* * *$ & $* *$ & $*$ \\
\hline TRP & 0.11 & 0.08 & 0.10 & 0.21 & 0.17 & 0.17 & 0.02 & $* *$ & ns & ns \\
\hline PHE & 0.21 & 0.26 & 0.29 & $1.97^{\mathrm{a}}$ & $1.62^{b}$ & $1.52^{b}$ & 0.06 & $* * *$ & $*$ & $* *$ \\
\hline ILE & 0.28 & 0.27 & 0.23 & $1.23^{\mathrm{a}}$ & $1.05^{b}$ & $1.04^{\mathrm{b}}$ & 0.03 & $* * *$ & $* * *$ & $*$ \\
\hline LEU & $0.49^{\mathrm{a}}$ & $0.36^{\mathrm{b}}$ & $0.34^{\mathrm{b}}$ & $1.92^{\mathrm{a}}$ & $1.21^{b}$ & $1.09^{b}$ & 0.08 & $* * *$ & $* * *$ & $* *$ \\
\hline LYS & 0.35 & 0.32 & 0.26 & $1.96^{\mathrm{a}}$ & $1.72^{b}$ & $1.64^{\mathrm{b}}$ & 0.04 & $* * *$ & $* *$ & $*$ \\
\hline PRO & $0.36^{\mathrm{a}}$ & $0.17^{\mathrm{b}}$ & $0.15^{b}$ & 0.38 & 0.33 & 0.33 & 0.02 & $* * *$ & $* * *$ & $*$ \\
\hline
\end{tabular}

nd, Not detected; ns, Not significant different $(p>0.05)$.

$* p \leq 0.05 ; * * p \leq 0.01 ; * * * p \leq 0.001$.

${ }^{\mathrm{a}-\mathrm{b}}$ Different letters in the same row indicate significant difference at $p \leq 0.05$. 
caused by the action of endogenous proteinases, oligopeptidases and aminopeptidases $\mathrm{C}, \mathrm{H}$ and $\mathrm{P}$ (Feidt et al., 1996; Moya et al., 2001). This accumulation of free amino acids is known to be important in the development of meat flavors (Toldra, 2006). An increase in particular amino acids caused by ageing has been found in the previous studies, representatively Parrish et al. (1969) and Field et al. (1971) found that large increases in alanine, glutamine, serine, valine, leucine and lysine are obtained by ageing of beef for $5 \mathrm{~d}$. Nishimura (1998) reported that rates increase in free amino acids of beef during the postmortem was $3 \mu \mathrm{mol} / \mathrm{g}$ meat per day, especially while large increases were in alanine, leucine, serine and valine. In addition, Migita and Nishimura (2006) suggested that amino peptidase D which newly-found distinct from amino peptidase B appears to contribute the increase in free amino acids, especially alanine and glutamine in meat during ageing.

In this study, only sulphur-amino acid like cystine was not significantly differed between ageing days. The cystine is not as essential because this can be made from methionine which is present but in a lower quantity about $0.17 \mu \mathrm{mol} / \mathrm{g}$ in $1 \mathrm{~d}$ aged beef. Furthermore, it is probably related to which Furst et al (1990) reported that cystine reproducibility and linearity were poor when using OPA or FMOC derivatives.

The higher homogenizing speed at $24,000 \mathrm{rpm}$ significantly affected the detection sensitivity of amino acids, also most amino acids tended to significantly increase with homogenizing speed however some amino acids such as serine, histidine, cysteine and proline showed a slightly increased in their mean values. Overall interactions between ageing and speed showed that significant differences for almost detected amino acids except for aspartic acid, serine, histidine, and tryptophan. This means that the mean values of amino acids increased with ageing time and big dispensing tool when high speed applied. The results suggested that the rotor turns at a high rpm; the tissues are rapidly reduced in size by a combination of extreme turbulence, cavitations and scissor like mechanical shearing occurring within the narrow gap between the rotor and the stator (Burden, 2008). Furthermore, mechanical disruption tissue generates by protein fragmenting and disrupting the sarcomere structure within myofibrils (Solomon et al., 2002). Major ultra-structural changes which occur during ageing are the disappearance of the transversal alignment of the Z-disks and sarcomere fragmentation (Feidt et al., 1996). This protein breakdown

Table 2. Changes in free amino acid contents during ageing, and by using smaller dispersing tool and different speed $(\mu \mathrm{mol} / \mathrm{g})$

\begin{tabular}{|c|c|c|c|c|c|c|c|c|}
\hline \multicolumn{9}{|c|}{ Smaller homogenizer } \\
\hline \multirow{2}{*}{$\begin{array}{l}\text { Amino } \\
\text { acids }\end{array}$} & \multicolumn{2}{|c|}{$1 \mathrm{~d}$} & \multicolumn{2}{|c|}{$7 \mathrm{~d}$} & \multirow{2}{*}{ SEM } & \multirow{2}{*}{ Ageing } & \multirow{2}{*}{ Speed } & \multirow{2}{*}{$\begin{array}{l}\text { Ageing } \\
\times \text { Speed }\end{array}$} \\
\hline & High & Slow & High & Slow & & & & \\
\hline ASP & nd & nd & 0.14 & 0.14 & 0.01 & $* * *$ & ns & ns \\
\hline GLU & 0.30 & 0.23 & 1.34 & 1.44 & 0.11 & $* * *$ & ns & ns \\
\hline ASN & 0.04 & 0.07 & 0.47 & 0.49 & 0.04 & $* * *$ & ns & ns \\
\hline SER & 0.30 & 0.32 & 1.31 & 1.07 & 0.08 & $* * *$ & ns & ns \\
\hline GLN & 0.90 & 2.25 & 4.66 & 6.80 & 0.43 & $* * *$ & ns & ns \\
\hline HIS & 0.14 & 0.07 & 0.38 & 0.43 & 0.45 & $* * *$ & ns & ns \\
\hline GLY & 0.40 & 0.35 & 1.07 & 1.01 & 0.11 & $* * *$ & ns & ns \\
\hline THR & 0.22 & 0.16 & 1.04 & 1.06 & 0.09 & $* * *$ & ns & ns \\
\hline ARG & 0.20 & 0.16 & 0.87 & 0.84 & 0.06 & $* * *$ & $\mathrm{~ns}$ & ns \\
\hline ALA & 4.40 & 3.61 & 10.31 & 11.14 & 0.97 & $* * *$ & ns & ns \\
\hline TYR & 0.17 & 0.15 & 1.22 & 1.02 & 0.08 & $* * *$ & ns & ns \\
\hline CYS & 0.12 & nd & 0.09 & 0.07 & 0.02 & $* * *$ & ns & ns \\
\hline VAL & $0.25^{\mathrm{a}}$ & $0.19^{b}$ & $1.09^{\mathrm{a}}$ & $0.82^{\mathrm{b}}$ & 0.06 & $* * *$ & $*$ & ns \\
\hline MET & 0.12 & 0.10 & 0.50 & 0.46 & 0.03 & $* * *$ & ns & ns \\
\hline TRP & nd & nd & 0.20 & 0.12 & 0.01 & $* * *$ & ns & ns \\
\hline PHE & 0.27 & 0.27 & 1.45 & 1.25 & 0.05 & $* * *$ & ns & ns \\
\hline ILE & 0.25 & 0.22 & $0.80^{\mathrm{a}}$ & $0.33^{\mathrm{b}}$ & 0.09 & $*$ & $*$ & $*$ \\
\hline LEU & 0.32 & 0.30 & 1.11 & 0.88 & 0.06 & $* * *$ & $\mathrm{~ns}$ & ns \\
\hline LYS & 0.25 & 0.23 & 1.22 & 1.18 & 0.08 & $* * *$ & $\mathrm{~ns}$ & ns \\
\hline PRO & 0.13 & 0.13 & 0.32 & 0.32 & 0.04 & $* *$ & ns & ns \\
\hline
\end{tabular}

nd, Not detected; ns, Not significant different $(p>0.05)$.

${ }^{*} p \leq 0.05 ; * * p \leq 0.01 ; * * * \leq 0.001$.

${ }^{\mathrm{a}-\mathrm{b}}$ Different letters in the same row indicate significant difference at $p \leq 0.05$. 
and polypeptide production degraded to small peptides and free amino acids. Therefore, postmortem ageing and homogenizing at high rpm treatment may improve the proteolysis and that the amino acids with high concentrations were released from degraded proteins which means that efficiently enzymes may work faster on smaller particles (Nishimura, 1998).

\section{Type and level of free amino acids as a function of} ageing and speeds of smaller dispensing tool

Most of the free amino acids which released by using the smaller dispersing tool (stator diameter $8 \mathrm{~mm}$, and rotor diameter $6.1 \mathrm{~mm}$ ) at high speed were slightly higher in their mean values when compared to those by using medium and slow speed but not significantly in $1 \mathrm{~d}$ and 7 $\mathrm{d}$ ageing samples (Table 2), only valine and isoleucine were significantly differed between homogenizing speed. Mean values also were not significantly differed when the interactions between ageing with homogenizing speed. Moreover, aspartic acid and tryptophan were not detected in $1 \mathrm{~d}$ ageing beef samples. Cystine also not detected in homogenized at lower speed. However, when the samples were aged for $7 \mathrm{~d}$, all amino acid appeared in both homogenizing speed (low and high). The absence of these two amino acids in the samples was also mentioned in the previous studies. Kivi (2000) reported that the tryptophan was lost when used ACN for deproteinization procedures. Furst et al. (1990) revealed that the aspartic acid and tryptophan were tough amino acids when used OPA reagent for derivatization. Moreover, aspartic acid is unstable and it is hydrolytic product of asparagines and this may be related with lower values of asparagines in lower speed. Smaller dispersing tool may also do not match to sample volume which became incomplete disruption of samples and resulted in lower amount of amino acids.

It has been established that homogenization at higher speed at 24,000 rpm is better for detecting an ageing effect on releasing amino acids than when samples were homogenized at medium and slower speeds by using an bigger dispersing tools. The several experiments revealed that the homogenization speed effected to the sample state. Hopkins et al. (2004) reported that the homogenization increases up to $15,000 \mathrm{rpm}$, the myofibrillar fragmentation index (MFI) values decreases compared to those obtained at slower speed such as $5000 \mathrm{rpm}$. Karumendu et al. (2009) revealed that the homogenization at $16,000 \mathrm{rpm}$ provided the greatest ability to detect ageing differences for particle sizes between samples aged for 1 and $5 \mathrm{~d}$ and rate MFI values.
Furthermore, using big dispensing tool for homogenizing the both $1 \mathrm{~d}$ and $7 \mathrm{~d}$ ageing samples at high speed, result indicated the mean values of all 20 detected amino acids were higher compared to those homogenized by the small dispensing tool. This might probably related to homogenization process, the use of bigger dispersing tool effectively destroyed tissue for amino acid releases and produced a much finer particulate due to physical breakdown of protein, plasma membranes, also physical disruptions of the myofibrillar ultra structure and intermyofibril linkages. Furthermore, incomplete sample disruption due to smaller dispersing tool may resulted in the reduction
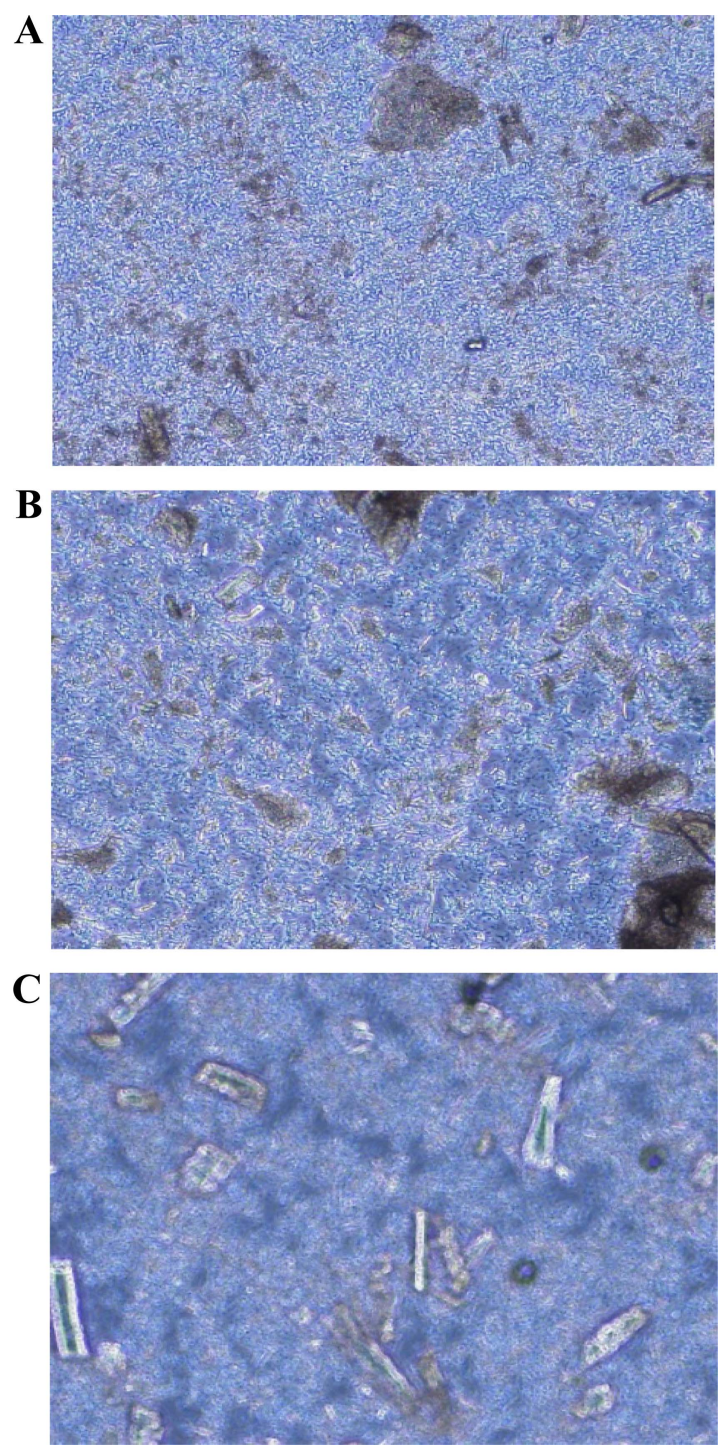

Fig. 1. Microstructure of the homogenized muscle sample at different speeds using bigger Rotor-stator homogenizer with S25 NK 19 G dispersing tools; A, 24,000 rpm; B, 19,000 rpm; C, 11.000 rpm. Using electronic microscope at 40x magnifications, coupled to a digital camera (Olympus, CKX 41, and Foculus IEEE1394, Germany). 

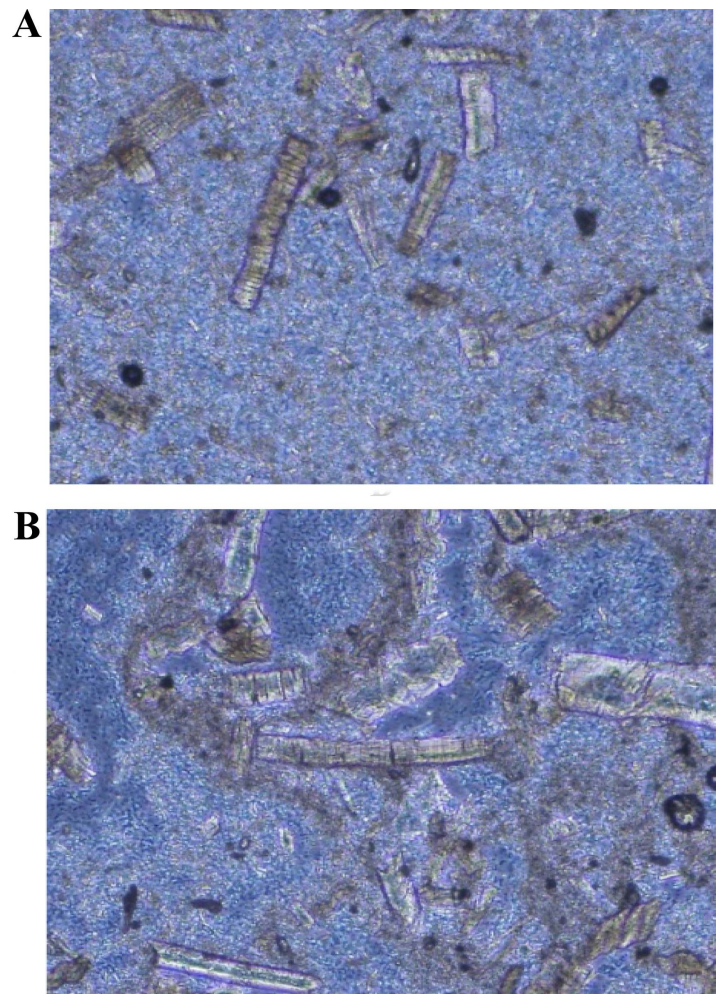

Fig. 2. Microstructure of the homogenized sample at different speeds by using smaller Rotor-stator homogenizer with S8 N8G dispersing tools; Homogenized A, 13.000 rpm; B, 11.000 respectively. Using electronic microscope at 40x magnifications, coupled to a digital camera (Olympus, CKX 41, and Foculus IEEE1394, Germany).

yields significantly and application of smaller dispersing tools may reduce the sample volume (Halpine, 2004).

\section{The microstructures of muscle fiber fragments for homogenized samples}

The microstructures of muscle fiber fragments of samples formed by homogenizing using two different dispensing tools at various speeds are showed in Figs. 1 and 2. It shows that more tissue disruption formed by using the big dispersing tool compared to the small one. The muscle fiber fragment size reduced when raising the homogenizing speed. Similarly, Hopkins et al. (2004) reported that homogenization speed affect the size of myofibrillar fragment and examined that myofibrils for homogenized at lower speed the myofibrils were much longer and still connected. It is logical to assume that the rotor stators quickly sheared samples at the high speed, reduced the muscle mass to fragments which were further reduced in size by subsequent homogenization steps. The big dispersing tool was effective in disrupting tissue for amino acids. Probably, there are the main reasons making the mean values and detection sensitivity of amino acids higher in the samples which were homogenized by big dispensing tool and high speeds (Tables 1 and 2).

\section{Conclusion}

Ageing of beef produced a significant increase in the mean values of free amino acids, which are important indicators relating to the eating quality such as tenderness, flavor and juiciness. Using big dispensing tool for homogenizing beef samples generated more number of amino acids and its mean value than using the small dispensing tool. Increase in homogenizing speed up to $24,000 \mathrm{rpm}$ produced higher mean values of amino acids and analytic sensitivity. The current data indicated that utilization of a big dispensing tool at high homogenization speed is the better condition for releasing free amino acid contents in beef sample as comparing between ageing time samples in terms of consistent and repeatable outcomes. As a technical note, the current study emphasized that a standard method for analyzing free amino acid types and levels of aged beef samples is very important for consistent results and compatible data with other laboratories.

\section{Acknowledgements}

It should be acknowledged that this work was supported by the research grants for the FTA issue project (No. PJ907055), RDA and the research grants for the FTA issue project (No. PJ008525), RDA, Republic of Korea.

\section{References}

1. Aristoy, M. C. and Toldra, F. (1991) Deproteinization technique for HPLC amino acid analysis in fresh pork muscle and dry cured ham. J.Agric. Food Chem. 39, 1792-1975.

2. Aristoy, M. C. and Toldra, F. (2009) Amino acids; Handbook of muscle foods, Nollet, L. M. L. and Toldra, F. (ed) CRC Press, pp. 11-30.

3. Burden, D. W. (2008) Guide to the homogenization of biological samples, Random Primers. 7, 1-14.

4. Feidt, C., Petit, A., Bruas-Reignier, F., and Brun-Bellit, J. (1996) Release of free amino acids during ageing in bovine meat, Meat Sci. 44, 19-25.

5. Field, R. A., Riley, M. L., and Chang, Y. O. (1971) Free amino acid changes in different aged bovine muscles and their relationship to shear values. J. Food Sci. 36, 611-612.

6. Furst, P., Pollack, L., Graser, T. A., Godel, H., and Stehle, P. (1990) Appraisal of four pre-column derivatisation methods for the high performance liquid chromatography determination of free amino acids in biological materials. J. Chromatogr. 499, 557-569. 
7. Halpine, S. M. (2004) Amino acids analysis by HPLC: Encyclopedia of chromatography, Cazes, J. (ed) Marcel Dekker, Inc., NY, pp. 1-4.

8. Hopkins, D .L., Martin, L., and Gilmour, A. R. (2004) The impact of homogenizer type and speed on the determination of myofibrillar fragmentation. Meat Sci. 67, 705-710.

9. Hawng, I. H., Park, B. Y., Cho, S. H., Kim, J. H., and Lee, J. M. (2004) Meat quality of highly manbled imported bef with reference to Hanwoo. Kor. J. Anim. Sci. Techol. 46, 1-8.

10. Kivi, J. T. (2000) Amino acids: Food analysis by HPLC, Nollet, L. M. L. 2nd ed, New York, pp. 55-83.

11. Migita, K. and Nishimura, T. (2006) Purification and characterization of $\mathrm{Cl}^{-}$activated aminopeptidase from skeletal muscle. Biosci. Biotechnol. Biochem. 70, 1110-1117.

12. Moya, V. J., Flores. M., Aristoy, M-C., and Toldra, F. (2001) Pork meat quality affects peptide and amino acid profiles during the ageing process. Meat Sci. 58, 197-206.

13. Nishimura, T. (1998) Mechanism involved in the improvement of meat taste during postmortem aging. Food Sci. Tech- nol. Int. Tokyo 4, 241-249.

14. Parrish, Jr., F. C., Goll, D. E., Newcomb, W. J., de Lumen, B. O., Chaudhry, H. M., and Kline, E. A. (1969) Molecular properties of post-mortem muscle. Changes in nonprotein nitrogen and free amino acids of bovine muscle. J. Food Sci. 34, 196-202.

15. SAS (2007) SAS/STAT Software for PC, Release 9.0, SAS Institute Inc., Cary, NC, USA.

16. Solomon, M. B., Liu, M. N., Patel, J. R., Bowker, B. C., and Sharma, M. (2002) Hydrodynamic pressure processing to improve meat quality and safety. Advanced technologies for meat processing. Nollet, L. M. L. and Toldra, F., (ed), pp. 219-244.

17. Woodward, C. and Henderson, J. W. (2007) High-speed amino acid analysis on 1.8ìm Reversed-Phase (RP) columns. Agilent technologies, Pharmaceuticals and Food 1-13.

(Received 2012.2.7/Revised 2012.3.22/Accepted 2012.4.2) 